\title{
Collaborative Approaches to Undergraduate Research Training: Information Literacy and Data Management
}

\author{
Hailey Mooney \\ W. Aaron Collie \\ Shawn W. Nicholson \\ Marya R. Sosulski
}

\begin{abstract}
The undergraduate research experience (URE) provides an opportunity for students to engage in meaningful work with faculty mentors on research projects. An increasingly important component of scholarly research is the application of research data management best practices, yet this often falls out of the scope of URE programs. This article presents a case study of faculty and librarian collaboration in the integration of a library and research data management curriculum into a social work URE research team. Discussion includes reflections on the content and learning outcomes, benefits of a holistic approach to introducing undergraduate students to research practice, and challenges of scale.
\end{abstract}

Keywords: Undergraduate research, research data management, faculty librarian collaboration, social work education

Undergraduate education provides students with foundational skills and abilities permitting entry into the workforce or advanced study at the graduate level. Introduction to research methodologies is an important aspect of the social work curriculum preparing students to base real world practice on the critical appraisal of various techniques and interventions. Research opportunities for social work students, such as those provided by undergraduate research experience programs, allow students to build relationships with faculty mentors and experience first-hand the research process, thereby amplifying critical thinking skills and preparing students to be producers (rather than just consumers) of professional knowledge (Moore \& Avant, 2008).

In today's data-rich research environment, a key component of these foundational skills is the ability to successfully navigate the organizational and technological aspects of research data production. The library and information science profession is contributing to the training of this new arena of research skills known collectively as research data management. In many cases, the integration of data and information skills is not explicitly part of the undergraduate research experience. By collaborating with librarians, faculty mentors for undergraduate research can significantly enhance the experience and build foundational skills for students giving them a leg up from their peers in the competition they will face for jobs or graduate school entry after graduation.

Hailey Mooney, MLIS, is the Data Services Coordinator and Social Sciences Librarian, W. Aaron Collie, MSLIS, is the Digital Curation Librarian, and Shawn W. Nicholson, MS and MSLIS, is the Associate Director for Digital Information, all with the Michigan State University Libraries. Marya R. Sosulski, Ph.D., is an Associate Professor in the School of Social Work at Michigan State University in East Lansing, MI. 
This paper will introduce readers to the importance of research data management skills to social work education and the role of librarians in providing instruction and support in this area through the lens of enriching the undergraduate research experience. A case study of faculty/librarian collaboration with an undergraduate research team will provide an example of a data management curriculum. This article fills a needed gap in addressing a life-cycle approach to research data management in the growing literature on undergraduate research experiences (URE) while demonstrating the relevance to social work education. Our experience of designing and implementing research data management modules and the reported authentic experiences of the students signals the potential benefit of integrated and detailed research data management instruction as routine for URE.

\section{Literature Review}

\section{Major Context: Research Data Management}

Due to the widespread adoption of technology in higher education, a new baseline requirement for many research projects is digitally managing the data and digital ephemera that support scholarly dissemination. Digital research data vary widely due to the irregular makeup of research itself, including within disciplines like social work where both qualitative and quantitative methods may be employed. Variations in project size, research methods, disciplinary norms, available resources, and expected outcomes combined with the many possible ways to represent information digitally result in a variegated landscape of research data. Because of this heterogeneity, research data requires equally nuanced responses for management, storage, preservation, and access. This problem in itself is not new and researchers have been pioneering methods of organizing, managing, analyzing, and collaborating around digital research data for over three decades.

Recent policy developments, such as the 2011 requirement from the National Science Foundation (NSF) to include data management plans in applications for funding, have pushed research data management into the spotlight of the scholarly communication crisis. Many funding agencies have followed suit to the original NSF impetus and seek to expand the reach of research dollars by recommending that research data be made available as an additional output of publically funded research. This, combined with the more general call for open and increased access to the results of research, has created an environment where the sharing of research data itself (in addition to publications based on that data) will increasingly be expected (Goben \& Salo, 2013). Largely in response to these changes, an effort to create scalable and standardized research data training, best practices, and services has resulted in the confluence of a number of extant disciplines (information science, research administration, higher education administration, information technology) into an emergent community of digital data curators.

Broadly defined digital data curation is a term that reflects a holistic approach for managing digital assets for their entire "lifecycle" of utility for research and scholarship. This includes selection, collection, analysis, interpretation, description, preservation, transformation, maintenance, access, long term archiving, and reuse. While digital 
curation is a term that is prevalent in the information science literature, in practice the strategies and actions which support the holistic model of data stewardship are often simplified and colloquial. Research data management and digital data curation are somewhat synonymous, with the former implying a streamlined research-centric lifecycle and the latter implying a more inclusive content agnostic lifecycle. This paper discusses data within the context of academic research in social work and therefore relies on the terminology of research data management.

It is suggested in a number of recent studies that the general knowledge and skills necessary to manage research data are not currently a major part of undergraduate, graduate, and doctoral curriculums (Carlson, Fosmire, Miller, \& Sapp Nelson, 2011; Scaramozzino, Ramirez, \& McGaughey, 2012). Carlson et al. identified in their assessment of faculty interviews that "[data management] skills, knowledge, and training needed by graduate students" was a "common theme" and that "Typically, faculty determined graduate students were unprepared to manage or curate the data effectively..." (p. 636). Those same faculty implied that there was a causality dilemma as "...they often could not provide adequate guidance or instruction because it was not an area that they knew well or fully understood" (p. 636). Scaramozzino et al. found a similar dilemma in surveying the College of Science and Mathematics at California Polytechnic State University; fully half $(50 \%)$ of respondents reported they were "not sure or not confident in their data management skills" and were "open to increased educational activities on the topic" (p. 360). Because researchers themselves widely vary in their command and comfort of information technology, it is unclear if research data management education can be consistently implemented by merely enforcing a new curriculum.

Despite these deficiencies, research data management is an expected job skill for graduate assistants, doctoral researchers, and faculty researchers (Carlson et al., 2011). If our next generations of researchers do not possess the baseline knowledge, skills, and experience that are in demand for contemporaneous research data management, we risk exacerbating the deficiency by producing an underprepared workforce.

The prerequisite knowledge for this baseline requirement has been positioned as a literacy that Carlson et al. (2011) term "data information literacy." They argue that data information literacy is a synthesis of related literacies that aim to foster understanding of key research concepts including methods of data collection or acquisition, data representation, data interpretation, statistical analysis, data manipulation, data management and preservation, and data summarization and presentation among other related skill sets.

\section{Why is Research Data Management Important to Undergraduate Research Experience Participants?}

Undergraduate research assistants are involved in meaningful research engagements with their faculty mentors. These beginning researchers through formal and at times funded undergraduate research experiences (URE) participate in various activities such as literature reviews, qualitative and quantitative data acquisition, coding, interpretation, and 
the presentation of analyzed results. Faculty ask undergraduate research assistants to work directly with their data in one way or another; therefore students should be aware of best practices for data management.

Many commentators point to the Boyer Report on Reinventing Undergraduate Education (Boyer Commission on Educating Undergraduates in the Research University, 1998), as spurring the birth of cross-cutting undergraduate research experiences at American research universities (Fechheimer, Webber, \& Kleiber, 2011). Later, Kuh and Schneider's (2008) highly influential High-Impact Educational Practices singled out undergraduate research as important for formulating questions, honing observational skills and working with advanced technologies as a means to enhance student engagement and increase student success. The intervening decade has witnessed a spate of descriptive studies outlining design and implementation of URE programs (Buckley, Korkmaz, \& Kuh, 2008; Cuthbert, Arunachalam, \& Licina, 2012; Howitt, Wilson, Wilson, \& Roberts, 2010; Wilson, Martinez-Uribe, Fraser, \& Jeffreys, 2011). Social work education programs are included in the incorporation of undergraduate research into student learning opportunities, both within the context of regular coursework and field placements (Rubin, Valutis, \& Robinson, 2010), as well as supplemental URE mentored programs (Hughes, Ortiz, \& Horner, 2012). The need to engage undergraduate students in positive research opportunities is especially salient in the field of social work. As an applied helping profession, overcoming research anxiety is seen as an important goal (Adam, Zosky, \& Unrau, 2004; Maschi, Probst, \& Bradley, 2009).

Naturally, the numerous descriptive studies bred a desire to understand the impact of URE programs on the students as well as the domains. A wealth of empirically-based theoretical explorations of perceived benefits for students and institutions has emerged (Bauer \& Bennett, 2003; Kardash, 2000; Russell, Hancock, \& McCullough, 2007). Generally, these studies reveal that URE students feel positive about their experience, yet some concern has been raised whether research skills have been advanced. Responding to these concerns is a growing body of qualitative and quantitative assessment literature (Fechheimer et al., 2011; Gum et al., 2007; Lopatto, 2007) focusing on the learning outcomes (problem formulation, methodology application, lab techniques) of the undergraduate research experience. Yet absent from these studies is an explicit account of research data management instruction, however loosely articulated.

It is our assessment that data management and the broader scope of data information literacy, indeed even basic library research skills, are not widely perceived as explicit goals of participation in undergraduate research despite an overarching goal for the advancement of real world research experience. Ideally, the URE engages students in knowledge creation by participation in the processes of discovery through the conduct of academic research. The advancement of knowledge is an exciting prospect, but any good researcher knows that there are less glamorous aspects to the research process, such as data entry. In fact, these less glamorous aspects are often what the undergraduate is assigned to do. But the glue that ties these mundane tasks to the glory of a well-received publication is the planning and execution of best practices in handling the information and data that bolster the process of discovery. We must ensure that students learn that data management is more than just a set of skills, it is about engagement in the creation 
and preservation of knowledge. As librarians, we often see students who are embarrassed to admit that they do not understand how to conduct a literature review, let alone decipher a call number location. Faculty often assume that students come to them with these socalled basic skills, yet the reality is that these skills often fall through the cracks and are not a formal part of the curriculum.

\section{Faculty and Librarian Collaboration}

How then, do we accomplish the integration of the undergraduate research experience and the development of library and research data management competencies? It will require the infusion of multiple expertise. Faculty mentors are experts in their own research topics and methodologies and can guide students through the process of assisting with specific aspects of projects. However, since most faculty are not experts in the areas of information science and the accompanying data and information literacies, collaboration with librarian partners as additional mentors in the research process rounds out the undergraduate research experience.

While bringing a librarian into the classroom to instruct students on the skills needed to write research papers, or referring students needing extra assistance with compiling bibliographies to consult with a librarian are both common examples of faculty reliance on librarian expertise, faculty collaboration with librarians as partners in research teams is not as widely done. Traditional models of faculty/librarian collaboration focus on the classroom instruction environment (Shumaker, 2011). As experts in the literature and practice of their own research areas, it can be easy for faculty to overlook the value of librarian mentorship for initiation of the student researcher. Likewise, librarians are often focused on working with undergraduates within the context of course assignments. This results in a lack of attention paid to direct instruction from librarians within the undergraduate research experience.

Since undergraduate researchers are engaged in knowledge creation along with their faculty mentors, the experience presents a collaborative opportunity for librarians and faculty to advance student learning in a holistic, hands-on approach (Stamatoplos, 2009). Increasingly, librarians are actively expanding their roles beyond the traditional confines of the library building and the reference desk and in many cases are partnering as members of research teams (Allard, 2012; Brandt, 2010; Carlson \& Kneale, 2011; Dewey, 2004). Both research faculty and librarians have much to share and learn from each other on the path towards synthesis of domain knowledge with data management skills for more efficient and impactful research.

Opening the undergraduate research experience to a combined faculty/librarian mentorship exposes students to the complete research process; not only will students work on a particular aspect of the faculty's research project, they will also benefit from foundational skill-building in the areas of data and information literacy. This requires an ongoing collaborative partnership between faculty, librarian, and student as members of a research team. Accomplishing this requires an embedded model wherein faculty and librarian experience a give and take of expertise sharing and learning from one another. 
By integrating librarians into undergraduate research, faculty can provide opportunities for students to be coached on best practices for data management.

\section{Project Context}

\section{Case Study: Social Work Research Team}

At Michigan State University (MSU), a large midwestern research university, the URE is situated within the Office of the Associate Provost for Undergraduate Education. The MSU URE has entered into its second half-decade of existence and its design, implementation and assessment tracks closely to the development of the body of literature. In Spring 2012, approximately 575 students participated in the MSU URE, with $92 \%$ of surveyed undergraduate researchers reporting involvement as a contributing member of their mentor's research team ("MSU Undergraduate Research Survey," 2012). Students were involved in undergraduate research across 14 colleges within MSU, with varying work experiences in different areas and with different mentors. The URE culminates at the end of each academic year in the University Undergraduate Research and Arts Forum, an event showcasing undergraduate research efforts where students share their work by presenting short talks or poster presentations.

Within the MSU School of Social Work, mentored undergraduate research has been shown to positively support student learning and provide an overall beneficial experience (Hughes et al., 2012). In the 2011-2012 academic year a unique Social Work URE was developed for a group of undergraduate researchers by their faculty mentor in collaboration with a small team of librarians. In normal cases, Social Work faculty would usually work with just one librarian, the subject specialist librarian for Social Work. However, given the experimental nature of the pilot project and the need for a depth of overlapping areas of expertise across the library faculty, a "library team" was formed to ensure the highest level of partnership and service to the URE research team. The library team consisted of the Subject Specialist Librarian for Social Work (conveniently also working in a dual role as the Data Services Librarian), the Data Curation Librarian (digital technical expert), and the Associate Director for Digital Information (project visionary).

The undergraduate research students were given distinct independent responsibilities within a given project in order to "own" the work and gain more specialized expertise in a particular area of research. Students also worked collaboratively with the team, including attending biweekly research team meetings so that the group benefited from each other's experiences. It was at these meetings where librarians joined the research team as embedded participant observers and contributed the library and research data management curriculum.

This collective group of students, faculty mentor, and librarians (the library team) made up the complete URE research team. The functioning of the URE research team would allow for students to participate in the faculty mentor's own research as had been done in previous years, while at the same time serving as a pilot project for the infusion of a research data management curriculum led by the library team. 
The purpose of this collaboratively led undergraduate research team was multifold:

1. To prove librarian support to students and faculty in their routine work, thereby fulfilling the traditional librarian liaison role.

2. To reveal knowledge about and interest in research data management, by having the library team act as quasi participant observers.

3. To test the efficacy of the seminar approach to library and research data management instruction.

The URE research team for academic year 2011-2012 included students working on two projects: data analysis of minority women's experience with spirituality and mental health care, and conception and planning for a study of recovery within the context of community mental health services. The second project is a rare example of a project in which the student team joined at the very beginning of the process of designing a new study.

The faculty mentor felt as though it was a risk to involve students with very little experience at the outset, where they would be actively participating in the development of the research questions, methods, implementation, and analysis. While the mentor had ultimate decision-making power, she entrusted the process to the team as a whole. The results were overwhelmingly positive. The mentor provided formal instruction in theory and methods, and the students applied the knowledge and skills to the problem. The students' questions about the instruments (survey and focus group and interview protocols) were particularly useful, as well as the range of their suggestions from which solutions were chosen. The coordination of tasks was delegated, but all of the students took part in each assignment. This way, the students also learned about management of tasks, as well as discrete skills in reviewing literature, designing and modifying instruments, scheduling and conducting data collection, entering survey data, and transcribing focus groups and interviews. In the mentor's assessment, the process was more dynamic, creative, and progressed more quickly than she had expected. The project progressed from initial discussions of what the study should look like to implementation (i.e., data collection and management) in approximately one semester (16 weeks). The team approach provided students with the chance to experience a range of research tasks, including work on data gathered using both qualitative and quantitative methods.

\section{Curriculum}

The library and research data management curriculum covered topics spanning from across the entire scope of a research project, from literature review to post-project storage. While a unique curriculum was developed, it was built from a review of existing data management training programs, and integration of social work education competencies (Council on Social Work Education, 2012). Both of these curricular components are discussed below.

There are numerous resources available aimed at training researchers in data management competencies. These include web resources such as online tutorials aimed directly at researchers (e.g., EDINA and Data Library, University of Edinburgh, n.d.; 
Strasser, Cook, Michener, \& Budden, 2012; University of Essex, n.d.) and teaching examples and resources for instructors (e.g., Federation of Earth Science Information Partners, 2012; Qin, Small, \& D'Ignazio, n.d.; Strangeland et al., 2010). Piorun et al. (2012) have published a curriculum framework aimed directly at undergraduate and graduate students with seven modules covering data types and formats; contextual details and metadata; storage, backup, and security; legal and ethical considerations; data sharing and re-use; and data archiving and preservation planning. These modules map to a set of core competencies based on an inventory of related curricula and interviews with students about their data management practices. Carlson et al.'s (2011) exploration of data information literacy also yielded a similar list of core competencies with additions in the areas of data discovery and acquisition, data conversion, quality assurance, disciplinary cultures, analysis, and visualization. Many of these additional competencies reflect Carlson et al.'s broader conception of data literacy including active research skills as well as management considerations.

Relevant social work education core competencies include critical thinking, research informed practice, ethical practice, and social justice. Our approach to the URE and the data management curriculum emphasizes the placement of data management within the larger holistic research process. This is necessary in order for students to see how their particular assignments on the research team contribute to the development of scientific knowledge building. Ethical concerns regarding the collection and treatment of human subjects data necessarily infuse the curriculum. Social justice is raised not only as an outcome of research and practice, but as an issue integral to the scholarly communication system where public access to research findings are often limited by publication venues and the lack of open data. Students well trained in good research practice will in turn do good social work practice.

The modules developed for the URE students divide the elements of the research process logically in order to provide a fundamental structure that is helpful for beginning students without a background in research. Although initially developed as training tailored to the needs of a specific research team, the modules are based on a broader set of universal research skills that can be widely applied. Some research assistants may be reluctant to admit that they are unsure or do not understand the research process. Integration of this curriculum into the URE saves time by helping the faculty mentor identify where students are starting with their knowledge and skills without having to engage in unnecessary backtracking to discern problems. By providing a grounding in the research process, students are able to begin to develop intuition about the intricacies and nuances of conducting research.

The modules outlined below were delivered as short lectures interspersed with opportunities for discussion and reflection. Topics were presented in tandem with the needs of the research team activities so that as often as possible they could be directly related to the actual real-time workflow of the students' engagement with the faculty mentor's research. Therefore, the modules represent a series of thematic content areas rather than content covered during a single session. In practice, the library team contributed module content in a manner responsive to the ebb and flow of the entire research team's work, so that covering a single module might be split over multiple 
meetings. In this way, our hope was to engage the students in a web of content delivery and practice activities; each reinforcing each to maximize learning. Brown and Adler (2008) refer to this as "reversing the flow." Their claim is that formal curriculum works from the presumption that you first fill students with content before sending them out to engage in practice. He argues that best learning derives from practicing the content. It is within this spirit that the modules were developed, winding about and allowing for contemporaneous practice to drive content delivery. We offer the module themes and attendant core elements in detail in hope that they can be readily put into practice in other contexts.

\section{Curriculum Modules Outline}

Module 1: Introduction to Literature Management

Topics covered: Literature Management, Citations

Conducting literature reviews and becoming familiar with the relevant literature is an important component of research. This module familiarized students with basic concepts for literature management by introducing Zotero (George Mason University), a free and open source citation management tool. Starting with this module was a deliberate choice to introduce students to productivity and organizational tools before even beginning their literature reviews. Integrating a citation management tool into the process of the literature review initiates students into approaching research in a systematic way and provides a relatable medium for the introduction of concepts such as organization, management, and collaboration. The research team created a shared group library in Zotero where students could post articles and share annotations. This creates a space for students that demands the intellectual work of thematically categorizing articles using folders and keyword tags to begin the work of critically assessing the literature, building skills in critical thinking and laying the foundation for research-informed practice. Additionally, discussion of what it means for a software program to be open source provides an opportunity for students to consider the social justice issues inherent behind the movement for free, open, and community-supported digital products, as well as the advantages and disadvantages of using an open source tool in research.

\section{Module 2: Literature and Data}

Topics covered: Literature Review, Source Evaluation, Finding Data

The students in the research group had already been exposed to a guest lecture on literature searching from the social work librarian in their regular course schedule. Since a basic familiarity with using library resources was already established, it was only necessary to refresh and reinforce best practices for conducting literature reviews. This was accomplished by viewing a video tutorial (Smith et al., 2009) and going over steps and techniques in the literature review process. Students were given the opportunity to reflect on previous experience and ask questions. In order to emphasize the role of critical thinking in the process of choosing appropriate articles to review, the evaluation of sources was also discussed, using De Montfort University's Information Source 
Evaluation Matrix (Towlson, Leigh, \& Mathers, 2009) as a tool to define criteria for the inclusion of articles in student literature reviews.

Next, a transition towards focusing on data began by drawing out the integration of data in the scholarly literature. Students were asked to identify the source of data analyzed in their literature review articles. After introducing the concept of secondary analysis as a research methodology, students were asked to determine if researchers collected original data or used data collected by someone else, such as data from a major government survey. By identifying instances of data re-use, the value of documenting and sharing data is uncovered as an important component of research practice. This sets the stage for the remaining modules.

\section{Module 3: Planning for Project and Data Management}

Topics covered: Project Parameters (Tools \& Environment), File Plans, Naming

Conventions, Formats, Short Term Storage

The overarching message of this module was that the research process is a complex project which can benefit from detailed planning and resource provisioning. Highlighted in this module were the parallel lifecycles of research, scholarship, and data. Data was identified as a central resource and fuel of research which requires not only expert interpretation and analysis but also competent stewardship. This concept was enforced by examining the benefits of fundamental data management tactics such as developing file naming conventions, creating flexible and interpretable file plans and understanding the risks and advantages of file formats. Naming and organizing data to increase accessibility is a component of this module. The primary value of creating file plans and file naming protocols was demonstrated as a way to enhance the collaborative research process and provide ease of use. Creation of a system that is easy not just for the current team but also for others who may join in the future is important.

The significance of backing up data is generally accepted as good practice (three copies in three different places: original, external/local, and external/remote), but the majority of the team admitted to being inconsistent in this area. Lost data is a chief concern, but issues of confidentiality including storing data securely, managing access, and handling of data in use (e.g., processing, transcribing, using quantitative or mixed methods analysis programs such as SPSS and Dedoose) were also discussed. These issues are especially crucial in light of ethical concerns around private client data. Options specific to the faculty research project were explained, such as using a secure server with limited access to the research team, as well as being protected from public access. Discussion ranged from password protection to vigilance about the need to safeguard personal laptops used for research in public spaces and in living spaces (e.g., away from roommates or family members who might accidentally or personally view the data; locking the doors of rooms or cabinets where they keep their computers). The entire research team then weighed the benefits and potential drawbacks of the various security options and came up with a plan that worked for all of them, and first and foremost maximized the safety of the data. Finally, a distinction was made between project-term data storage and post-project term data archiving. 
Module 4: Data Collaboration and Sharing

Topics covered: Project Collaboration, Project Documentation, Metadata, Data Sharing

Collaboration and sharing around research data was introduced as a scalable practice that starts with strong project documentation. The recently covered topics of file plans and file naming conventions were highlighted as the type of documentation that can help establish an authoritative structure for data management. Guidelines for selecting course or content management systems, wikis, and other tools for collaboration around documentation were discussed with special attention given to version control and access control. Discussion centered on how good practices for capturing documentation for small team of researchers can be used as a building blocks to develop and capture more detailed description required for validation and reproducibility such as lab notebooks, research protocols, methodology, and metadata standards.

Collaboration and documentation were revisited at the end of the semester in order to create a final project documentation and data management plan that could be passed on to the next URE student group. This reinforced good research practice and an integrated view of individual student responsibilities into the whole of the research process. Importantly, providing the building blocks throughout the URE program for grounding in the research process, with a particular focus on data management for collaborative purposes, helped to provide students with ownership of the study and data. This built motivation for students to claim parts of the project and share their knowledge and expertise with the team and with the broader university community as part of their final project poster presentations.

\section{Module 5: Archiving and Reporting on Research Data}

Topics covered: Long Term Storage, Data Publishing, Data Citations, Data Presentation

A session on data presentation was delivered as students were preparing posters for presentation at the Undergraduate Research and Arts Forum, giving this topic a heightened sense of relevancy. A slide set was prepared with a series of examples of differing methods of data presentation from simple text-based descriptions such as lists and tables to more advanced visual-representations of data including charts, figures, illustrations, and visualizations. These examples were examined for their benefits or drawbacks by applying Tufte's (2001) guiding principles for design of quantitative data, Slone's (2009) guiding principles for presenting qualitative data, and Klass' (2008) principles for presenting social science data.

Data stewardship and curation was presented as the ongoing preservation of access and enhancement of data during its lifetime of utility. Long term or archival data storage was discussed as a baseline requirement for preservation of access to data and therefore differentiated from short or project-term data storage. The discussion circled back to confidentiality and ethical treatment of client data as students considered what types of data might be appropriate to archive and publish for other researchers to use, and what types of data would need to remain restricted or be eventually destroyed. Common methods of data archiving were examined including self-archiving, journal deposit and publication, and institutional and disciplinary repositories. Examples of each of these 
methods were discussed, with particular attention given to data citations, available metadata, and sustainability. Publishing data via the process of archiving was considered as a positive research practice in that it provides the ability for others to reproduce results or re-use data for new purposes. Although data archives such as the Inter-University Consortium for Political and Social Research have been around for more than 50 years, recent changes in the digital scholarly communication system have made data sharing within reach of many more research projects than before. The open access movement raises important social justice issues around the availability of research outside of the academic environment, especially in social work where many practice outside of the ivory tower.

\section{Results and Discussion}

\section{Student Reflections}

Our goal was for students to develop foundational skills and conceptual frameworks necessary to be conscientious researchers concerned about data management and positive contributors to the evolving system of scholarly communication and data-intensive research. We can share representative reflections of authentic experiences so as to frame future design, pedagogy, and assessment developments. These reflections are based on observation and discussion, as well as short written "minute papers" gathered throughout the duration of the program.

Throughout the curriculum, we asked students to draw from their existing knowledge and experience to create personal relevance to the material. There is nothing like the reality of putting data management best practices into action. This is especially salient when asked to reflect on current data backup strategies for course assignments. Student responses ranged from dedicated Dropbox accounts to haphazard use of flash drives, campus networks, and email accounts. One student intended to back up to an external hard drive, but was waiting on delivery of the appropriate connector cable from a family member. Asking students to consider their own personal data management practices drives home the point that planning and executing research data management best practices is an important component of a well-designed research project.

Another good example is file naming and organization practices. Students gravitated towards the practical advice that they could put directly into personal practice. Using descriptive file names with dates and versions was an epiphany that students saw as useful, making research data files accessible to a team and easily transferable to their own course work. Students noted the importance of organization as a key takeaway:

The most valuable thing I have learned from working with the library team this year would be with my organization skills. The library team has helped me to anticipate future research problems and how preparing for these hiccups will allow for a much smoother research process. A lot of the skills that I have learned have helped me in the classroom, especially with writing papers. 
Another student shared that one of the most valuable lessons she learned was "The importance of organization of data. This not only eases your own experience of analyzing the data but makes it accessible to others long after your own study is done."

At the end of the experience, students appeared to have gained a definite grasp of the research process and how it plays out in real life; the various components involved in planning and moving forward a project. One student's reflection demonstrates this outcome:

Working as an undergraduate research fellow gave me an inside understanding of the complex and iterative process that is foundational to academic research. From the initial stages of IRB approval to grant funding, facilitation, and the inevitable roadblocks, each stage in the research process is intricate. Just as most academic research is a conversation between past and present literature, the research process is less linear than it is circular; every phase of the research has the potential to affect a later step.

Students recognized that the skills they learned as undergraduate researchers are readily applicable to their future professional activities. In one case, a student felt that she was awarded a spot on a different research team "because of my experience with the library staff and experience on this project." She goes on to explain that the "skills we learned this year are easily transferable to any kind of research in all academic fields. I am going to be more prepared in all academic and professional senses because I am sensitive to things such as: what files to save things as, how to have multiple back-ups of my data, and how to stay organized on my own as I go rather than trying to catch up." Another student reflected that she is already putting her newfound knowledge to work at her new job where the need to

[think] critically about seemingly small tasks to maximize efficiency and ease of use has already crept in...I think the research process teaches students to analyze assumptions and seek out better modalities rather than accepting the status quo. I've learned to see inefficiencies and time-wasters that I had never noticed before, in addition to learning how to spot possible problems in order to preventatively troubleshoot, which will definitely be a valuable skill set as I continue with research.

A widening of perspective about research, both the process and its outcomes, was another notable reflection:

I have enjoyed the team aspect of our meetings. I see how effective it is to go beyond your department when needed and ask for support so that ultimately your research can become something that is useful to not only your team but to others interested in the same questions/findings.

The collaborative aspect of the project made an impression with this student, showing the understanding that research does not happen in a vacuum. This is an especially edifying reflection for the library team since it represents the successful integration of library resources and services into research and the awareness that research data (in addition to published articles) can be a valuable scholarly output. 
These reflections show that, through exposure to research combined with thoughtful discussions of research and data management best practices, students learned valuable practical skills and were able to see the larger context within which the scholarly research enterprise takes place.

\section{Faculty Mentor}

Working with many students at different levels, while rewarding, is time-consuming and can be resource-intensive. For this reason, some faculty members shy away from working with undergraduates. A research team needs to be able to work together, draw on each member's strengths, and produce results for a cogent analysis. The question of how one prepares students with little or no experience to move into tasks that require skills along the continuum of basic to advanced has not been resolved. To an extent, the process is idiosyncratic. However, this training curriculum crystallizes the essential parts of each step in the design and implementation of a research study. Having students work through the modules with the Library Team, along with individual consultations with them as needed, reduced the mentor's training load from weeks to days and the amount of work for the mentor shifted from a deficit to a net positive balance of training input and work output. According to the mentor:

The undergraduate research initiative funded by the university has increased my research capacity a great deal. Because I don't normally have funding to hire undergraduate research assistants, I rely on the URE program for extra hands. I hire, on average, three undergrad research assistants each year, and training can be onerous. Some students can start immediately doing literature reviews, joining in data collection events, data processing and management, and even analysis. Many, however, start at the beginning with little knowledge and few skills, and it may take a lot to get them up to speed. But all of them are expected to conform to my expectations and do the work thoroughly and well. Achieving economies of scale is essential to getting the work done effectively and efficiently, while giving the students the best experience possible.

Time spent in training helped the mentor understand where the students were starting with their knowledge and skills, assist them, and prevent errors. The mentor reflected:

Research supervisors often assume that research assistants-at this point, mostly younger people-understand how to work with data, because they have always been around technology. Also, it's second nature to us. We sometimes forget that we had to learn it, and how we learned it. In general, I think that because I don't always work on research in a linear way, it can be confusing for the students if I move from one piece of the study to another as quickly as I'm used to. But I realize that if I teach the research process in a systematic way, it's good modeling for the students.

The synergy of the curriculum with the actual work of the research projects enhanced student and faculty workflows. According to the mentor: 
Having [the Library Team] in on the whole process was essential to our success. As the PI, I could have made an executive decision. That might have been more expedient, but I would have had to consult with the Library Team anyway, since I didn't know all of the options and I value their input. So, hearing about the alternatives happened in the group with the students present. As always, the questions that the students asked added to the conversation, because I wouldn't have thought of things that would be most helpful for them as they worked together.

The mentor also appreciated that, as a practice, the team also held each other accountable for backing up data. This was achieved primarily through sharing tasks and making sure that updates and work completed were posted in the appropriate, secured places. The secure site where the raw and processed data were kept was monitored informally, and team members reminded each other to post their work regularly. The mentor began to think differently about questions of organization and documentation, as well:

Changing the way I thought about naming and organizing data files was difficult. Everybody has their preferred way of labeling that makes sense to them, but then expecting five to ten other people to be able to understand and use it the same way is somewhat unrealistic. It was good for me to be on "the other side" again, like I was as a graduate student in my first experiences as a research assistant. This [curriculum] gave me the chance to sit back and reflect on the logic of organizing schemes. Now, I have to learn about the rest of the team's way of thinking. I think what they came up with was better organized than what I had, and will be easier to explain to future cohorts.

What the mentor found is that when she asked the senior students to manage and delegate tasks to the junior team members, they were able to pick up the responsibility quickly because of their deeper understanding of the process. What normally would take a week or two to teach students was presented in one day, and the students then had each other and the library team for support.

For this particular undergraduate research experience, the University encouraged research assistants to develop their own research questions from the faculty mentor's study. The students identified unanswered questions in which they were interested, identified themes and/or variables in the data, analyzed them, and reported results and interpretation. The students presented their research findings as posters at a universitywide forum, where students represented every college and discipline. By the end of the year, the students were able to describe their projects in detail from beginning to end, including the roles they played in the research design. The guidance from the Library Team prepared them for answering questions about their work, especially those from the perspectives of people outside of the discipline.

Perhaps the most important aspect of the project is the collaboration because the students have learned when, where, and how to ask for help and where, when, and how to provide their opinions and understanding. Affirming this impression, the mentor reflected: 
I am confident that this group can join another project somewhere after they graduate, or design their own if they needed to, to evaluate their practice. I know they can do it, because I've seen them think through the process. They know what it takes to work together in a diverse group to problem-solve, to take a load of data and shape it into something meaningful, and to look at it from all sides.

In the end, the mentor found the close collaboration very effective for teaching the students the skills and knowledge they needed. The model of individual assistance is not practical, however, if the curriculum were to be applied to all of the undergraduate research assistants in the department. The important lessons that the mentor took from the experience centered on two points: 1) the connections made between the technical skills and conceptual frameworks are essential, and the expertise of the Library Team was invaluable for teaching the fundamentals; 2) the relationships among the research team members are critical to success. In practice, it is unrealistic to expect that each research team include a dedicated librarian/data specialist. But a close affiliation, through which the faculty and students have regular formal and informal contacts, offers substantial benefits. The challenge to will be to find the balance of instruction and guidance, on a scale that will be as inclusive as necessary. Future formats may include a program that could be open to any interested faculty mentors and undergraduate research assistants, consisting of prescribed workshops presented by a library team combined with periodic attendance by the librarians at team research meetings and individual consultations.

\section{Librarians}

As librarians do not typically play an involved role in research team meetings, a primary benefit of this collaboration is the opportunity for the library team to see behind the curtain and observe the structure and discourse of student and faculty collaboration during the undergraduate research experience. Faculty in turn were benefited by personal attention to their research support needs. Librarians and faculty have a symbiotic relationship: librarians simultaneously observe and participate in research and scholarship, aiming both to understand and support the scholarly enterprise. One of the librarians reflected on the value of this relationship:

The field of librarianship is changing along with evolution in the scholarly communication system and the focus on data-intensive research. The opportunity to have an inside look at the research process for a team at our university is invaluable as we are re-shaping our roles to meet changing needs. Studying how researchers do their work enables me to discern what current needs are and learn how to best meet those needs. Understanding research and informationseeking behavior is a really important aspect of my work so that I can purchase the right collections, learn how to provide the right guidance to new scholars, and advocate for investment in additional central university research infrastructure.

The modularized curriculum provided flexibility to enhance the faculty member's agenda. Material and delivery is prepared in advance of meetings, and could be presented or delayed depending on the progress of the project as well as the topics and issues raised 
during the meetings. A typical meeting balances between education, training, reporting and discussion of research and task progress. However, this degree of focus and attention is not easily scalable. Despite the modularized curriculum allowing for flexibility of delivery, the overhead of preparation and frequent meeting required by the embedded method is not particularly time efficient.

After acclimating to the dynamics of the group, it was possible to respond to teachable moments by providing immediate and tailored instruction. This instruction was provided in an ad hoc nature, and out-of-band with the regular presentation of the modularized curriculum. The corollary effect is the ability to prepare for upcoming content or revisit recently covered topics. This means that not only is it possible to assess student comprehension, but the library team is able to address gaps or correct misunderstanding as issues arise. One librarian reflects on the curriculum delivery:

At first it was difficult to realize that all of the material I had prepared could not realistically be delivered according to the calendar we had planned. Although our curriculum stressed that research is not always a linear process, I still like to plot out a plan, especially for instruction. But we were melding the two. It was very different from my usual instruction work, where I am in and out of the classroom in one session and have to carefully plan a detailed lesson that covers everything in one fell swoop. In the end, I actually grew to enjoy the development of an ongoing relationship that allowed for curriculum to be delivered over a period of time in concert with real world needs.

This dynamic was also mutually beneficial for the library team. The iterative nature of instruction provided an accelerated feedback loop for the library team to improve and expand upon earlier topics. Many of the improvements discovered during this method have since been applied to subsequent revisions of the modules. Because very few curricula exist that were designed for undergraduate comprehension, this situation provided a valuable test of content appropriateness. Another unanticipated but noted benefit of embedded delivery of content is the close tandem of the lifecycle model for data management and the research process as a whole. Information gathering, planning, management, analysis, reporting, and publication are paralleled in these curricula providing a serendipitous synergy of research agenda and traditional library instruction.

Because those with knowledge of best practices for research data management often are neither the same as those with knowledge of reference and instruction nor the same as those with detailed knowledge of the research process, team instruction diffuses teaching and learning. The social work librarian reflects on this aspect of the project:

The experience of integrating recently conceived content on data information literacy along with our well-established information literacy competencies has proven to be valuable. A current struggle for many librarians is how to add data management work on top of their existing responsibilities. I see now that this is a continuum of service, which helps make data management work more accessible. Working with my colleagues as a team has helped me develop a better understanding of the digital curation concepts that are used to inform the work of data management. 
Resulting is a forum for shared learning: librarians grow more adept at advocating support for tools (e.g., workflow software) and services (e.g., digital storage) for university researchers; researchers are better equipped to pursue data-centric improvements to research efficiency and impact; data managers are initiated into the research process and empowered with new teaching methods; and students are elevated into new roles on the research team for which they were previously underprepared.

\section{Conclusion}

As a case study of a pilot project, this experience has laid the groundwork to explore the integration of a library and research data management curriculum into the undergraduate research experience. There is room for further assessment of student skills and knowledge within the context of mentored undergraduate research specific to this integrated skill set. In addition, the reflective assessments point up challenges of scale, time commitment, and the need for multiple areas of domain knowledge. The question of how to move forward in a way that provides the greatest impact for more students still remains unanswered. Given that the undergraduate research experience involves many small distinct teams of faculty mentors and students working on different projects, there may not be a way to replicate the embedded model beyond one or two research teams. Reaching the majority of research teams led by social work faculty may mean the forfeiting of a deeply personalized experience and teaching model for a more generic set of workshops that will apply across the board as a baseline of library and research data management competencies. Although this would lose the high level of synergy and reciprocity between faculty, librarian, and student along with the direct relevance that is gained from mapping the curriculum directly to the agenda of a particular research project, it would still serve as an opportunity to build foundational skills for students and open their eyes to the holistic research process beyond the individual tasks to which they may be assigned. This launching pad effect could still have considerable utility and benefits as part of the overall mentored research experience.

However, without the knowledge gained from the experience of working together over the course of a year as a collaborative faculty/librarian/student team, the potential for greater impact and the efficacy of the curriculum would never have been realized. This experience developed awareness, enhanced understanding, and built capacity for the research team in regards to workflow and data management, and for librarians in regards to researcher needs and behavior. It is clear that simple modules relating good practice resonate with an undergraduate population. Librarians, in particular, bring a unique insider/outsider perspective that allows for the imparting of expertise across the lifecycle of a research project which is beneficial to both students and faculty. Our project revealed that students possess a need and are excited to learn and situate their contributions to faculty research within a holistic context encompassing processes and best practices for the conduct of scholarly research in general. Drawing out and directly addressing the skills, knowledge, and best practices surrounding library and research data management serve to make explicit what is often an implicit area of scholarly practice. Mentored undergraduate research is an opportunity to expose students to the research process from 
soup to nuts and a forum for faculty and librarians to contribute their expertise for their personal mutual benefits as well as for the enhancement of the student experience.

\section{References}

Adam, N., Zosky, D. L., \& Unrau, Y. A. (2004). Improving the research climate in social work curricula. Journal of Teaching in Social Work, 24(3-4), 1-18. doi:10.1300/J067v24n03_01

Allard, S. (2012). DataONE: Facilitating eScience through collaboration. Journal of eScience Librarianship, 1(1). Retrieved from http://escholarship.umassmed.edu/jeslib/vol1/iss1/3

Bauer, K. W., \& Bennett, J. S. (2003). Alumni perceptions used to assess undergraduate research experience. The Journal of Higher Education, 74(2), 210-230.

Boyer Commission on Educating Undergraduates in the Research University. (1998). Reinventing undergraduate education: A blueprint for America's research universities. Stony Brook, NY: Author. Retrieved from ERIC website: http://files.eric.ed.gov/fulltext/ED424840.pdf

Brandt, D. S. (2010). Librarians, advocacy, and the research enterprise. In W. C. Welburn, J. Welburn, \& B. McNeil (Eds.), Advocacy, outreach \& the nation's academic libraries: A call for action (pp. 43-54). Chicago, IL: Association of College and Research Libraries.

Brown, J. S., \& Adler, R. P. (2008). Minds on fire: Open education, the long tail, and learning 2.0. EDUCAUSE Review, 43(1), 16-32.

Buckley, J. A., Korkmaz, A., \& Kuh, G. D. (2008, April). Student-faculty research: Priming the pump for additional student-faculty contact. Paper presented at the meeting of the American Educational Research Association, San Diego, CA. Retrieved from http://cpr.iub.edu/uploads/priming the pump.pdf

Carlson, J., Fosmire, M., Miller, C. C., \& Sapp Nelson, M. (2011). Determining data information literacy needs: A study of students and research faculty. portal: Libraries and the Academy, 11(2), 629-657.

Carlson, J., \& Kneale, R. (2011). Embedded librarianship in the research context: Navigating new waters. College \& Research Libraries News, 72(3), 167-170.

Council on Social Work Education. (2012). Educational policy and accreditation standards. Retrieved from http://www.cswe.org/File.aspx?id=13780

Cuthbert, D., Arunachalam, D., \& Licina, D. (2012). "It feels more important than other classes I have done": An "authentic" undergraduate research experience in sociology. Studies in Higher Education, 37(2), 129-142. doi:10.1080/03075079.2010.538473

Dewey, B. I. (2004). The embedded librarian. Resource Sharing \& Information Networks, 17(1-2), 5-17. doi:10.1300/J121v17n01_02 
EDINA and Data Library, University of Edinburgh. (n.d.). Research data MANTRA [Online course]. Retrieved from http://datalib.edina.ac.uk/mantra/

Fechheimer, M., Webber, K., \& Kleiber, P. B. (2011). How well do undergraduate research programs promote engagement and success of students? CBE Life Sciences Education, 10(2), 156-163. doi:10.1187/cbe.10-10-0130

Federation of Earth Science Information Partners. (2012). Data management short course. ESIP Federation Wiki. Retrieved from http://wiki.esipfed.org/index.php/Data_Management_Short_Course

George Mason University, Roy Rosenzweig Center for History and New Media. Zotero [Computer software]. Retrieved from http://zotero.org

Goben, A., \& Salo, D. (2013). Federal research: Data requirements set to change. College \& Research Libraries News, 74(8), 421-425.

Gum, A., Mueller, K., Flink, D., Siraj, S., Batsche, C., Boothroyd, R., \& Stiles, P. (2007). Evaluation of a summer research institute in behavioral health for undergraduate students. The Journal of Behavioral Health Services \& Research, 34(2), 206-218. doi:10.1007/s11414-007-9059-1

Howitt, S., Wilson, A., Wilson, K., \& Roberts, P. (2010). "Please remember we are not all brilliant": Undergraduates' experiences of an elite, research-intensive degree at a research-intensive university. Higher Education Research \& Development, 29(4), 405-420. doi:10.1080/07294361003601883

Hughes, A., Ortiz, D. V., \& Horner, P. (2012). Academic, professional, and community outcomes of a faculty-mentored BASW research experience. Journal of Baccalaureate Social Work, 17, 133-147.

Kardash, C. M. (2000). Evaluation of undergraduate research experience: Perceptions of undergraduate interns and their faculty mentors. Journal of Educational Psychology, 92(1), 191-201. doi:10.1037/0022-0663.92.1.191

Klass, G. M. (2008). Just plain data analysis: Finding, presenting, and interpreting social science data. Lanham, MD: Rowman \& Littlefield Publishers.

Kuh, G. D., \& Schneider, C. G. (2008). High-impact educational practices: What they are, who has access to them, and why they matter. Washington, DC: Association of American Colleges and Universities.

Lopatto, D. (2007). Undergraduate research experiences support science career decisions and active learning. CBE-Life Sciences Education, 6(4), 297-306. doi:10.1187/cbe.07-06-0039

Maschi, T., Probst, B., \& Bradley, C. (2009). Mapping social work students' perceptions of the research process: A qualitative follow-up study. Journal of Baccalaureate Social Work, 14(2), 63-78.

Moore, L. S., \& Avant, F. (2008). Strengthening undergraduate social work research: Models and strategies. Social Work Research, 32(4), 231-235. 
MSU Undergraduate Research Survey. (2012, Fall). UR@MSU: Newsletter for MSU Faculty about Undergraduate Research, (1). Retrieved from http://urca.msu.edu/files/userfiles/file/Faculty\%20Newsletters/Fall\%202012\%20New sletter.pdf

Piorun, M., Kafel, D., Leger-Hornby, T., Najafi, S., Martin, E., Colombo, P., \& LaPelle, N. (2012). Teaching research data management: An undergraduate/graduate curriculum. Journal of eScience Librarianship, 1(1). Retrieved from http://escholarship.umassmed.edu/jeslib/vol1/iss1/8

Qin, J., Small, R., \& D'Ignazio, J. (n.d.). The science data literacy project: Educator resources. The science data literacy project. Retrieved from http://sdl.syr.edu/?page id=15

Rubin, D., Valutis, S., \& Robinson, B. (2010). Social work education and student research projects: A survey of program directors. Journal of Social Work Education, 46(1), 39-55.

Russell, S. H., Hancock, M. P., \& McCullough, J. (2007). Benefits of undergraduate research experiences. Science, 316(5824), 548-549. doi:10.1126/science.1140384

Scaramozzino, J. M., Ramírez, M. L., \& McGaughey, K. J. (2012). A study of faculty data curation behaviors and attitudes at a teaching-centered university. College \& Research Libraries, 73(4), 349-365.

Shumaker, D. (2011). Beyond instruction: Creating new roles for embedded librarians. In Embedded librarians: Moving beyond one-shot instruction (pp. 17-31). Chicago: Association of College and Research Libraries.

Slone, D. J. (2009). Visualizing qualitative information. The Qualitative Report, 14(3), 488-497.

Smith, E., Duckett, K., Bankston, S., Classen, J., Orphanides, A., \& Baker, S. (2009). Literature reviews: An overview for graduate students [Video tutorial]. North Carolina State University Libraries. Retrieved from http://www.lib.ncsu.edu/tutorials/lit-review/

Stamatoplos, A. (2009). The role of academic libraries in mentored undergraduate research: A model of engagement in the academic community. College \& Research Libraries, 70(3), 235-249.

Strangeland, E., Lloyd-Smith, L., Peano, I., Lazar, S., Young, G., \& Jeffrey, S. (2010). DataTrain project. Cambridge University Library. Retrieved from http://www.lib.cam.ac.uk/preservation/datatrain/index.html

Strasser, C., Cook, R., Michener, W., \& Budden, A. (2012). Primer on data management. DataONE. Retrieved from http://www.dataone.org/sites/all/documents/DataONE_BP_Primer_020212.pdf 
Towlson, K., Leigh, M., \& Mathers, L. (2009, Winter). The information source evaluation matrix: A quick, easy and transferable content evaluation tool. SCONUL Focus, 47, 15-18.

Tufte, E. R. (2001). The visual display of quantitative information ( $2^{\text {nd }} \mathrm{ed}$.). Cheshire, CT: Graphics Press.

University of Essex. (n.d.). Create and manage data: Training resources. UK Data Archive. Retrieved from http://www.data-archive.ac.uk/create-manage/trainingresources

Wilson, J. A. J., Martinez-Uribe, L., Fraser, M. A., \& Jeffreys, P. (2011). An institutional approach to developing research data management infrastructure. International Journal of Digital Curation, 6(2), 274-287. doi:10.2218/ijdc.v6i2.203

\section{Author note}

Address correspondence to: Hailey Mooney, Data Services Coordinator and Social Sciences Librarian, Michigan State University Libraries, 366 W. Circle Drive, East Lansing, MI 48824. Email: mooneyh@msu.edu 